\title{
Modelo de alineación estratégica para una vinculación social efectiva
}

\author{
Marcelina Martínez-López \\ Instituto Tecnológico y de Estudios Superiores de Monterrey \\ marcelina martinez@hotmail.com \\ Gustavo Parada Sarmiento \\ Instituto Tecnológico y de Estudios Superiores de Monterrey \\ gaps96@gmail.com \\ Gabriel Madriz Cano \\ Instituto Tecnológico y de Estudios Superiores de Monterrey \\ gabriel.madriz@gmail.com
}

\begin{abstract}
Resumen
En este proyecto se realizó un ejercicio de alineación estratégica para el área de vinculación social de una institución educativa de nivel superior, se logró identificar los activos tangibles e intangibles y la clasificación de los mismos, creando así el sistema de capitales del área. Dichos capitales fueron evaluados y ayudaron a determinar los puntos fuertes y débiles en vinculación social, y esto permitió alinear dichos capitales proponiendo estrategias que coadyuven en el cumplimiento de los objetivos particulares del área. Los resultados del estudio son propuestas de líneas de acción a corto, mediano y largo plazo, por ejemplo la generación de nuevas funciones enfocadas a actividades que generan mayor valor al área, porque están enfocadas en el logro de los objetivos. Con este proyecto se muestra la aplicabilidad del modelo de alineación estratégica para escuelas de nivel superior, particularmente en el área de vinculación social.
\end{abstract}

\section{Palabras claves}

Vinculación social, alineación estratégica, sistema de capitales, instituciones educativas.

\section{Introducción}

La importancia del conocimiento para la competitividad de las compañías, organizaciones e incluso economías es ampliamente aceptada. Según Mertins, Heisig y Vorbeck la administración del conocimiento es parte de la cultura corporativa de una organización, la cual apoya el intercambio de información, conocimiento y experiencias entre empleados y departamentos, es la suma de procedimientos que determinan la generación, distribución y aplicación de conocimiento para lograr metas organizacionales (2003).

Carrillo (2001), refiere que la administración del conocimiento es el resultado de una visión holística de lo que es valioso en la organización y que permite la capitalización integrada de los diferentes tipos de conocimiento. Carrillo (2002) lo define como una estrategia para identificar, sistematizar y desarrollar el universo de los capitales de la 
organización. La administración del conocimiento permite encontrar, entender y usar el conocimiento para generar un valor, mediante la utilización de propuestas sistemáticas, esta requiere tres procesos clave: administración de sistemas de capitales, administración de capital humano y administración de capital instrumental.

Este proyecto está enfocado en la aplicabilidad del sistema de capitales de Carrillo como parte de la metodología aplicada en una materia de maestría, el proyecto es realizado el área de vinculación social del Instituto Tecnológico de Monterrey, Campus Monterrey. Vinculación social está dividida en Atención a Organizaciones, Gestión y Evaluación de Proyectos Sociales y Monitoreo de proyectos. Cuyo propósito es promover redes y vínculos entre alumnos de servicio social e instituciones, a partir de iniciativas y oportunidades, que traducidas a proyectos y programas sociales propician una experiencia que contribuye a fortalecer las competencias ciudadanas del alumno y a mejorar situaciones en desventaja en las áreas de educación, cultura, empleo, desarrollo de capacidades e infraestructura comunitaria. Dicha área requería hacer una restructuración de actividades y puestos pero enfocada en los procesos clave y que generan mayor valor al área y saber si estos se estaban realizando efectiva y eficientemente, con el propósito de mejorar y fortalecer aquellos que son más importantes para el logro de los objetivos del área.

El objetivo de este proyecto fue realizar un ejercicio de alineación estratégica para el área de vinculación social, identificando los activos tangibles e intangibles de la misma, con el fin de clasificar los activos en diversas categorías, creando así el sistema de capitales del área basado en el modelo de sistema de capitales de Carrillo (2002), dichos capitales identificados y clasificados fueron evaluados con instrumentos que se elaboraron para lograr establecer el valor de cada capital, esto con el fin de identificar la brecha entre el valor real y el valor ideal de cada uno, obteniendo así las bases para proponer líneas de acción que ayudarán a disminuir dicha brecha.

La importancia de identificar el sistema de capitales radica en ayudar a determinar los puntos fuertes y débiles de la institución educativa en cuanto a vinculación social, así como alinear dichos capitales para ofrecer estrategias que coadyuven en la formación de estudiantes como ciudadanos con conciencia social, con capacidad analítica y con un sentido crítico, lo anterior permite establecer el "servicio social ciudadano" (De Gortari, 2007). Los resultados del estudio muestran las áreas de oportunidad de vinculación social y con base en ellos se proponen líneas de acción a corto, mediano y largo plazo, por ejemplo, la generación de nuevas funciones enfocadas a actividades que generan mayor valor al área.

\section{Modelo de Sistema de Capitales}

Carrillo propone un enfoque de administración de sistemas de capitales que está basado en identificar lo que es valioso para la organización (2002), este modelo fue el utilizado porque es parte de la metodología de una materia de maestría, el modelo es mostrado en la figura 1, a partir de este se fue colocando en cada categoría cada uno de los elementos valiosos del área de vinculación social, este modelo fue desarrollado en un entorno empresarial, por lo cual resulta 
relevante para las organizaciones productivas. Gracias a su base de valor genérico, su aplicabilidad a organizaciones no productivas es cada vez más evidente. El sistema de capitales basado en conocimiento ha sido aplicado a unidades de investigación y desarrollo (CKS, 1998;
Torres, 2002), universidades (Barrientos, 2002) y en el gobierno (Villarreal, 2002). Para la identificación de capitales del Departamento de Vinculación Social, se utilizó el Sistema Genérico de Capitales, como parte de un ejercicio educativo.

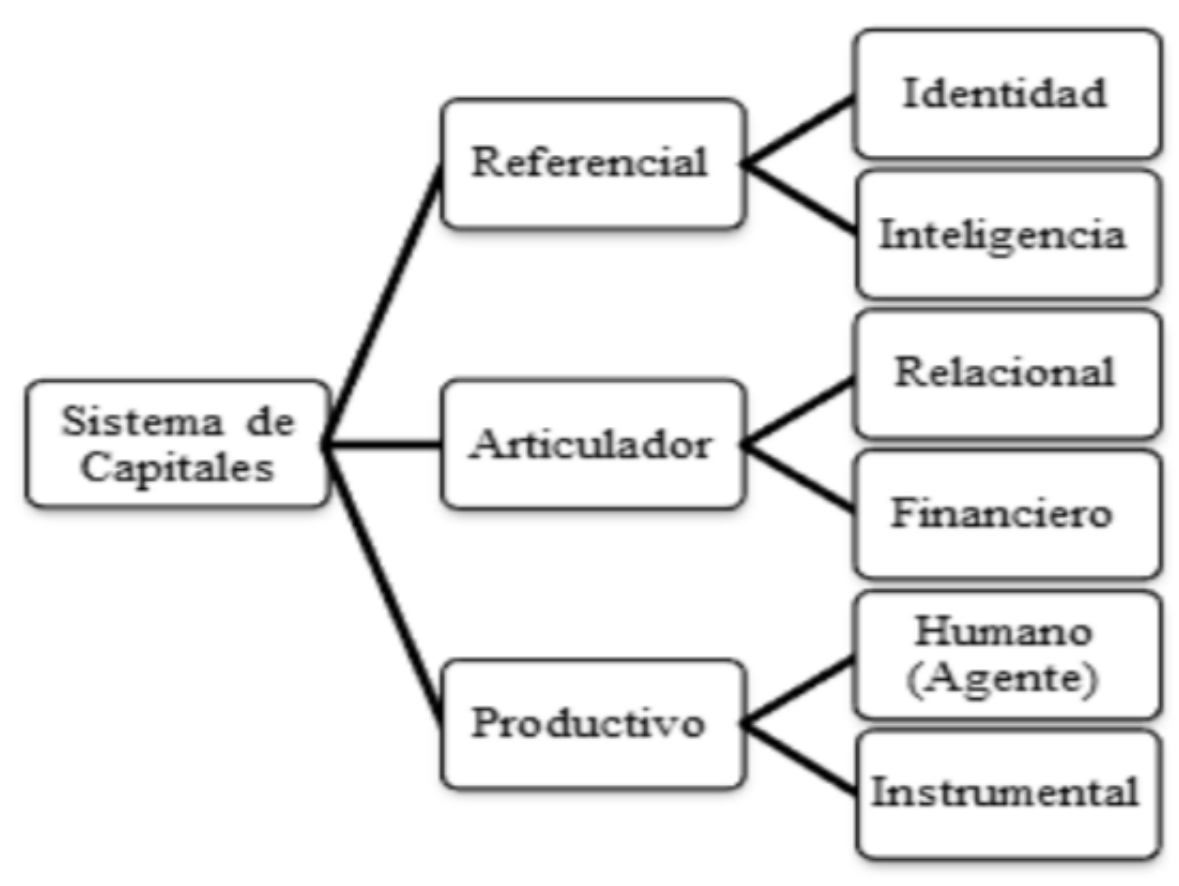

Figura 1. Sistema de capitales. Adaptado de Carrillo (2002).

Para explicar la taxonomía de capitales de este modelo a continuación se presenta la definición de cada uno de ellos:

Capital referencial: Son los elementos valiosos que permiten identificar y alinear todos los elementos valiosos de la empresa. Se subdivide en el capital identidad y en el capital inteligencia.

- Identidad: Es aquel que define los rasgos y características de la organización, puede ser la forma en cómo es percibida la empresa desde afuera (Identidad Externa) y cómo se define a nivel interno (Identidad Interna). La identidad le brinda claridad y diferenciación a la empresa, este se desarrolla a partir de la habilidad de la empresa para poder formar una imagen propia y controlable de sí misma para poder dar a conocer lo que esta quiere decir de si y la manera en la que es percibida por su entorno.

- Inteligencia: Este permite la respuesta oportuna a eventos y entidades externas, con el fin de adquirir conocimiento externo para mejorar las condiciones internas.

Capital Articulador: es el que contiene los elementos valiosos que sirven para interrelacionar 0 intercambiar otros elementos de valor. Está compuesto por el capital financiero y el capital relacional. 
- Financiero: Es la expresión monetaria de algunos o todos los elementos de valor, indica su viabilidad económica a largo plazo. Se refiere al capital tradicional con el que las organizaciones han valuado habitualmente a sus activos tangibles.

- Relacional: Este involucra la relación de la organización interna y externamente. El externo es aquel que involucra la manera en la cual la organización se puede relacionar con los agentes fuera de la misma, y el interno hace referencia a la comunicación y relación en diferentes niveles jerárquicos, entre los departamentos y dentro de los mismos. Se refiere al valor que posee $y$ produce una organización como resultado de su interacción con cuatro agentes del entorno: clientes, proveedores, aliados y competidores, que en su conjunto forman la red de relaciones que mantiene la organización con su exterior.

Capital productivo: Este involucra a todos los elementos de valor de la organización que participan en el proceso de producción. Comprende el capital humano y el instrumental.

- Humano: Se refiere a las capacidades y competencias de generación de valor aportadas por individuos dirigidos a mejorar el desempeño es decir son aquellos comportamientos de las personas relacionadas con sus capacidades de generar y compartir nuevo conocimiento.

- Instrumental: son los medios de producción a través de los cuales los otros capitales aprovechan su capacidad de generar valor. Pueden ser tangibles o intangibles como procesos, prácticas, indicadores, tecnologías, etc.

\section{Metodología}

La metodología utilizada en la realización de este proyecto permite obtener una perspectiva integral de como la alineación estratégica y el conocimiento permiten maximizar la oferta distintiva de valor de una organización, con tres objetivos específicos: definir el sistema de capitales, establecer los términos de alineación y proponer iniciativas de administración de conocimiento. Dicha metodología está integrada por cuatro etapas:

- Análisis del contexto de la organización: es la información interna y externa de la organización para conformar un marco de referencia sobre el área de vinculación social, incluyendo el giro, misión, visión, valores, estrategias, organigrama, procesos, clientes, proveedores, etc.

- Definición del sistema de capitales y sus indicadores: se refiere al desarrollo del sistema de capitales del área seleccionada, así como sus indicadores. Esto incluye la estructura de capitales, glosario de capitales, ponderación de capitales con base $100 \%$, nombre y definición de los indicadores de los capitales de la organización.

- Medición y alineación: el objetivo de esta etapa es diseñar la estrategia e instrumentos de medición de los indicadores, por capital de la organización, valuar los capitales e identificar la brecha entre el valor ideal del capital y el valor real. Esto incluye la tabla descriptiva de los capitales, con el 
valor ideal, el valor real y la brecha, así como el diagrama radial y su interpretación.

- Líneas de acción: en esta etapa se definen las líneas de acción para el desarrollo de los capitales de la organización, con el objetivo de cerrar las brechas detectadas, a nivel estratégico, de capital humano y de capital instrumental. Dentro de esta metodología se establece que dichas líneas de acción deben cumplir con ciertas especificaciones como ser factibles, que promuevan la innovación y también deben contener la información necesaria para su aplicación como es nombre, descripción, capitales a los que se enfoca, tiempo que se requiere para su implementación, personas o roles involucrados y los beneficios que se lograrán con la aplicación de la misma.

La aplicación de esta metodología en la materia de alineación estratégica y conocimiento del negocio permite visualizar y aplicar el sistema de capitales del negocio, así como reconocer los procesos básicos de la administración del conocimiento.

\section{Resultados}

Los resultados de este trabajo se presentan en este artículo en el orden de la metodología expuesta anteriormente. Se realizó el análisis del contexto del área de Vinculación Social la cual pertenece al Departamento de Formación Social y Apoyo a la Comunidad, ubicado dentro del Instituto Tecnológico y de Estudios Superiores de Monterrey, Campus Monterrey, obteniendo que el principal objetivo del área es crear vínculos y redes con instituciones sin fines de lucro, ofreciendo la oportunidad a alumnos del tecnológico a prestar un servicio social que le contribuya a fortalecer sus propias competencias ciudadanas y a crearles un sentido social. También se documentó la misión, valores, estrategias, procesos, los actores involucrados y principales funciones de las personas que integran el área.

A partir del análisis, así como de la observación y entrevistas realizadas al personal del área, y con base en el modelo de capitales de Carrillo, se logró recopilar la información necesaria para realizar el sistema de capitales del área mostrado en la tabla 1.

Tabla 1. Capitales del área de vinculación social.

\begin{tabular}{|c|c|c|}
\hline 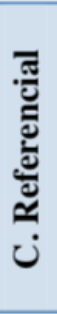 & $\begin{array}{l}\text { Capital Identidad } \\
\text { - } \text { Misión } \\
\text { - Visión } \\
\text { - Valores } \\
\text { - Imagen de sitio web } \\
\text { - Estructura organizacional } \\
\text { - } \quad \text { Cultura organizacional }\end{array}$ & $\begin{array}{l}\text { Capital Inteligencia } \\
\text { - Conocimiento externo } \\
\text { - Prácticas de vinculación social } \\
\text { - Calidad del servicio }\end{array}$ \\
\hline ن & $\begin{array}{l}\text { Capital Relacional } \\
\text { - Socios formadores } \\
\text { - Departamento de Formación y Servicio } \\
\text { Social } \\
\text { - Departamento de Desarrollo Estudiantil } \\
\text { - Lideres estudiantiles }\end{array}$ & $\begin{array}{l}\text { Capital Financiero } \\
\text { - Nómina } \\
\text { - Presupuesto Semestral }\end{array}$ \\
\hline
\end{tabular}




\begin{tabular}{|c|c|c|}
\hline & - Organizaciones afines & \\
\hline 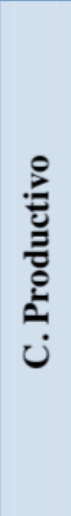 & $\begin{array}{l}\text { Capital Humano } \\
\text { - Competencias organizacionales del socio } \\
\text { formador } \\
\text { - Competencias del alumno para } \\
\text { participación } \\
\text { - Competencias del alumno formado } \\
\text { - Competencias estratégicas del área }\end{array}$ & $\begin{array}{l}\text { Capital Instrumental } \\
\text { - Semáforo de corresponsabilidad } \\
\text { - SIASS (Aplicación de Gestión) } \\
\text { - } \text { Sinio Web } \\
\text { de proyectos } \\
\text { - Políticas de compromiso con socios } \\
\text { - } \text { formadores } \\
\text { - } \text { Especificaciones de relación } \\
\text { - Infraestructura física } \\
\text { - Medios de Comunicación }\end{array}$ \\
\hline
\end{tabular}

Al sistema de capitales se le asignó una ponderación considerando el nivel de contribución individual de éstos en el cumplimiento de las estrategias del área de Vinculación enfocadas a la formación ciudadana del alumno y el desarrollo social, asignándosele así un valor ideal a cada uno de los capitales.
Debido a que el total de capitales era una cantidad muy grande para asignarles indicadores, diseñar y aplicar instrumentos de medición, se tomó la decisión de seleccionar aquellos que resultaron con una ponderación alta en comparación con los demás capitales como se muestra en la tabla 2.

Tabla 2. Ponderación de los capitales de vinculación social.

\begin{tabular}{|c|c|c|c|c|c|}
\hline \multirow{2}{*}{$\begin{array}{c}\text { Tipo } \\
\text { Referencial }\end{array}$} & \multirow[t]{2}{*}{ Subtipo } & \multirow[t]{2}{*}{ Capital } & \multicolumn{3}{|c|}{ Ponderación (\%) } \\
\hline & & & & & 37 \\
\hline & \multirow[t]{7}{*}{ Identidad } & & & 16 & \\
\hline & & Misión & 4 & & \\
\hline & & Visión & 3 & & \\
\hline & & Valores & 3 & & \\
\hline & & Imagen de sitio web & 1 & & \\
\hline & & Estructura organizacional & 2 & & \\
\hline & & Cultura organizacional & 3 & & \\
\hline & \multirow[t]{4}{*}{ Inteligencia } & & & 21 & \\
\hline & & Conocimiento externo & 5 & & \\
\hline & & Prácticas de vinculación social & 8 & & \\
\hline & & Calidad del Servicio & 8 & & \\
\hline \multirow[t]{2}{*}{ Articulador } & & & & & 29 \\
\hline & Relacional & & & 24 & \\
\hline
\end{tabular}




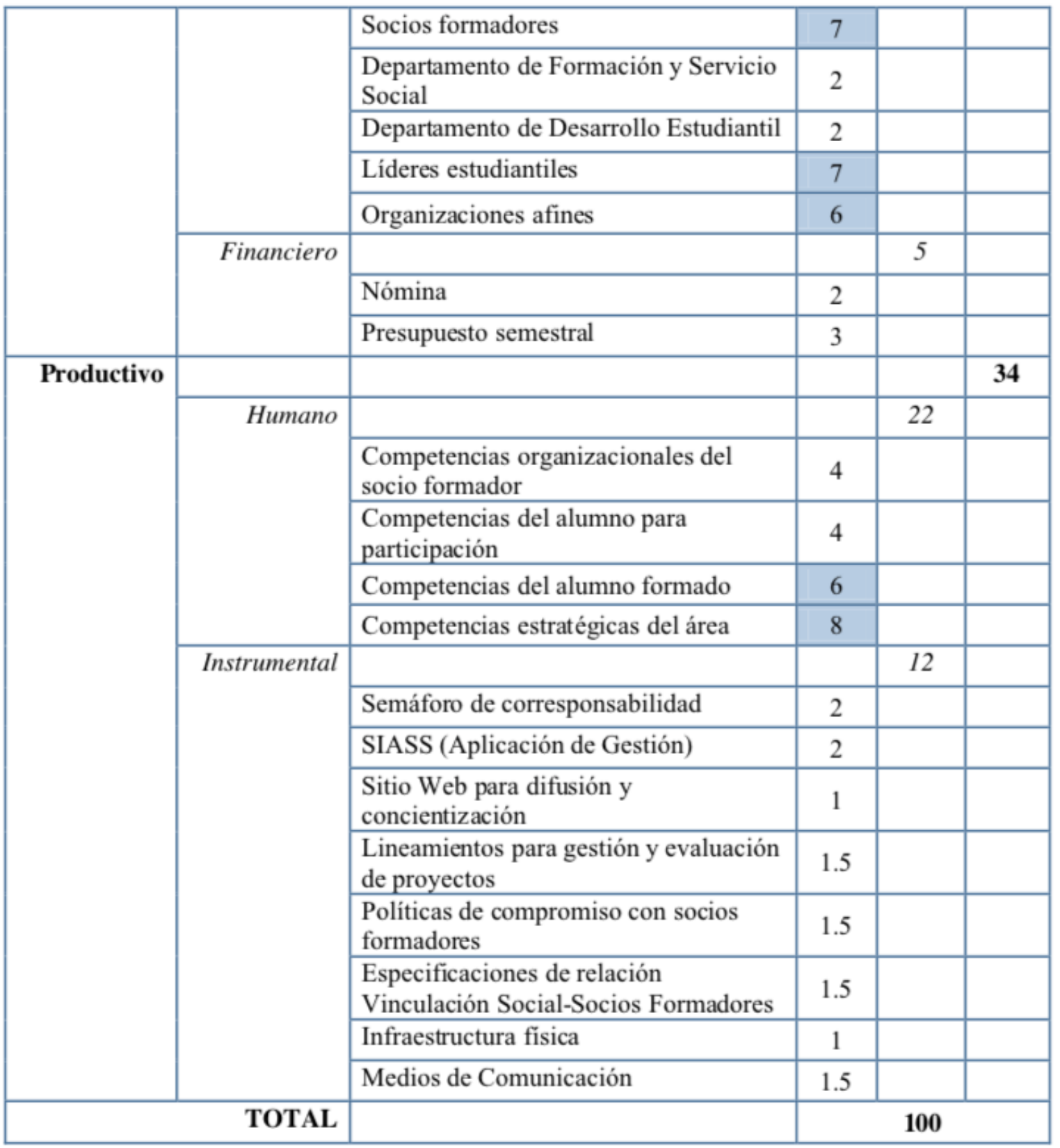

Los capitales seleccionados fueron siete Prácticas de Vinculación Social, Calidad del Servicio, Socios Formadores, Líderes Estudiantiles, Organizaciones afines, competencias del alumno formado y Competencias estratégicas del área. Estos representan el $50 \%$ del valor de la ponderación total del sistema. Para poder obtener el valor real de cada uno se diseñaron los indicadores para cada capital seleccionado y se aplicaron cuestionarios para cada tipo de involucrado en los procesos principales de esta área como se muestra en la figura 2, los tipos de involucrados son alumnos que cursan o cursaron el servicio social, las instituciones no lucrativas que reciben a los alumnos de servicio social del Tecnológico de Monterrey y las personas que laboran en el área. 
Figura 2. Encuesta aplicada a socios formadores (instituciones no lucrativas).

\title{
Encuesta de Evaluación para Socios Formadores
}

\begin{abstract}
Buen dia.
La presente encuesta es elaborada por Estudiantes de Maestría del ITESM.

Estas preguntas son parte de un Proyecto de la materia de Alineación Estratégica y Conocimiento del Negocio, con el objetivo de identificar puntos fuertes y áreas de mejora del área de Vinculación Social. La meta es dar una retroalimentación y diagnóstico que beneficie al área, los alumnos y las Organizaciones Socio Formadoras, como la suya.

Los siguientes preguntas ayudarán a evaluar rasgos clave para este estudio, con el fin de verificar el nivel de conocimientoy aplicación de los mismos en el área de Vinculación Social. Conteste con toda sinceridad.

Agradecemos que esta encuesta sea contestada a la brevedad, no le tomara más de 10 minutos.
\end{abstract}

En relación al Departamento de Vinculación Social

¿Cómo califica...?

Excelente Muy Buena Regular Mala

[1] Satisfacción con la relación. [2]La calidad en los BENEFICIOS a la Sociedad con la ayuda recibida

[3]Relación del Perfll de los alumnos asignados

con el trabajoen sus proyectos

\begin{tabular}{|l|l|l|l|}
\multicolumn{2}{l}{ Buena } & \\
\hline & & & \\
\hline & & & \\
\hline & & & \\
\hline
\end{tabular}

[4] ¿A qué sector beneflcia su institución principalmente?

\begin{tabular}{|l|l|l|l|}
\hline$\square$ Artey Cultura & $\square$ Educación \\
\hline Asesoria Especializada & $\square$ Empredimiento \\
\hline Clenciay Tecnología & $\square$ Infraestructuray Desarrollo Humano \\
\hline$\square$ Discapacidad & $\square$ Valoresy Desarrollo Humano \\
\hline Ecologia y Medio Ambiente & Otra. especifique & \\
\hline
\end{tabular}

Considera que su Institución...

\begin{tabular}{|l|l|l|l|l|}
\hline$[6] 2$ Ha realizadosugerencias al área de & & & \\
Vinculación. Social? & & & & \\
\hline $\begin{array}{l}\text { [7]_Considera que las sugerencias que ha hecho al } \\
\text { área de Vinc. Social, han sido puestas en práctica? }\end{array}$ & & & & \\
\hline$[8] 2$ Retroalimenta alos alumnos? & & & \\
\hline
\end{tabular}

[9] ¿Podria listar brevemente los beneficios obtenidos en su institución como Socio Formador? (E). Mejora en logística: Enseñanza de Idiomas, Mejoras de hábitos, otros)

[10] ZIdentifica usted áreas de oportunidad para el área de Vinculación Social? Si (zcuales?) No

A partir de los resultados obtenidos de la aplicación de los instrumentos se definieron los valores actuales de cada capital. Lo anterior permitió identificar las diferencias que existen entre la ponderación que se le dio a cada uno de los 
capitales en función del valor que aportan al área de vinculación y el valor esperado (ponderación), el valor real de los capitales evaluados se muestra en la figura 3.

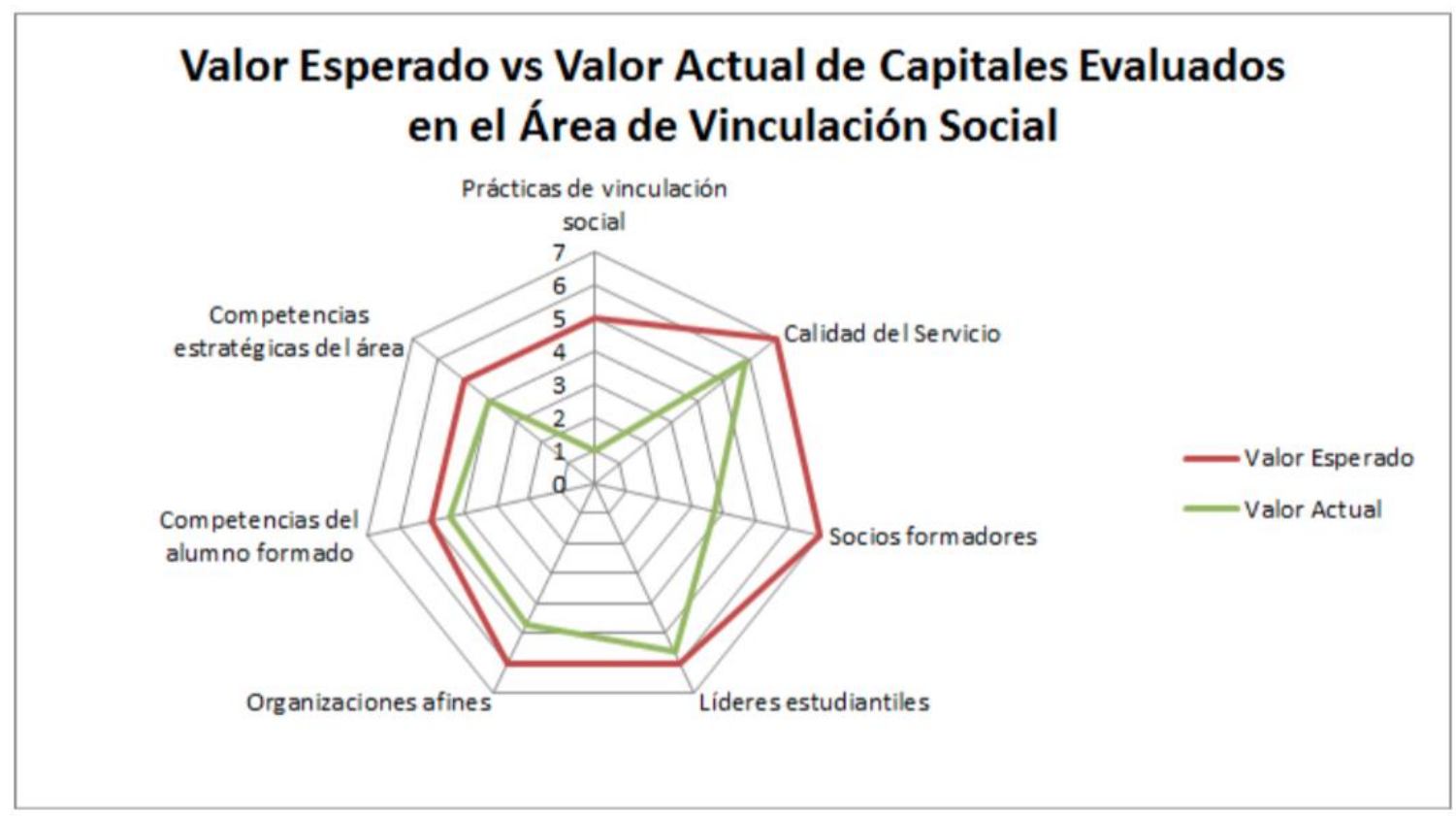

Figura 3. Valor esperado vs valor actual de capitales evaluados en el área de Vinculación social. Derivado del valor real y valor esperado surge la brecha que permite identificar aquellos capitales que aportan gran valor al área y que no fueron valorados positivamente, lo cual era uno de los objetivos principales de esta investigación. A partir de estas brechas se identificaron dos capitales con grandes oportunidades de mejora (Prácticas de Vinculación Social de tipo inteligencia y Socios formadores de tipo relacional), para los cuales se crearon estrategias y líneas de acción, con el fin de desarrollarlos y enriquecerlos. Las líneas de acción son estrategias a seguir que impulsan el incremento de los capitales evaluados con la finalidad de mejorar las áreas claves de la organización. Estas recomendaciones tienen el fin de reducir las brechas existentes y potenciar la capacidad de generación de valor en el área de Vinculación Social.

El formato para presentar las líneas de acción al responsable del área de vinculación social cumplió con los requerimientos de información para su completa y exitosa aplicación.

La primera línea de acción propuesta está enfocada en que el área participe de forma activa y programe la asistencia a eventos como congresos relacionados con servicio social, y que se establezca contacto con el área de vinculación social de otras universidades para compartir experiencias y adquirir prácticas valiosas que se puedan adaptar en su contexto, adquiriendo así capital inteligencia, específicamente aumentando el valor de los capitales "Prácticas de vinculación social" y "organizaciones afines".

La segunda línea de acción está enfocada a mejorar la satisfacción de los Socios Formadores (son las instituciones donde los alumnos realizan el servicio social) en la relación con Vinculación Social. Esta propuesta se puede capitalizar en dos formas una es mejorar la relación directamente con los representantes de las 
instituciones tomando en cuenta sus propuestas y sugerencias y otra es mejorar la relación con los Socios Formadores mediante estudiantes de servicio social, donde estos sean una extensión del área de Vinculación Social. Mejorando la relación con las instituciones a través de la escucha activa de sus intereses o propuestas de mejora. Esta línea de acción aumenta el capital relacional en específico "Socios formadores".

Con esto se logró la alineación del sistema de capitales la cual consiste en establecer el estado actual en el que se encuentra el área de Vinculación Social como punto de referencia, $y$ establecer los objetivos a los cuales se desea llegar, acercándose lo más posible a un estado ideal para el área.

\section{Discusión de resultados}

Estas líneas de acción propuestas permitieron al área de vinculación social conocer sus procesos y actividades clave, las cuales les generan mayor valor y a partir de estas se logró definir nuevas funciones y puestos, es decir hacer la reestructuración pero en base a los capitales que son de mayor peso para el área. Algunas de las líneas de acción propuestas fueron aplicadas de forma inmediata, pero otras quedaron pendientes porque requerían la autorización y recursos de niveles jerárquicos más altos. El alcance de esta investigación llega hasta el planteamiento de las líneas de acción y no la corroboración de la correcta aplicación y éxito de las mismas, así como tampoco la buena disposición y colaboración de las personas que se encarguen de hacer las actividades de mejora.

En primera instancia los resultados

de este proyecto permiten al departamento de Vinculación social tomar decisiones respecto a la prioridad de proyectos para lograr los objetivos organizacionales. Adicionalmente el modelo propuesto ofrece oportunidad a otros departamentos académicos de plantear esquemas similares que muestren sus activos y que les permitan validar su alineación con respecto a los objetivos institucionales, en los cuales estos departamentos juegan un rol relevante.

En este ejercicio fueron valoradas las líneas de acción en función de su impacto en el cumplimiento de los objetivos organizacionales, así mismo el ejercicio realizado incluye un sistema de indicadores que facilita la medición del cumplimiento de los objetivos organizacionales. En otras palabras, el sistema de indicadores además de medir la brecha entre el valor actual e ideal de los capitales, conforma una herramienta para dar seguimiento al cumplimiento de las estrategias propuestas, precisamente para cerrar esas brechas.

\section{Referencias}

Barrientos, X. (2002). Diseño de una estrategia de desarrollo universitaria basada en sistema de capitales. Disertación Doctoral, UV, Tecnológico de Monterrey.

Carrillo, F. J. (2001). Meta-KM: A Program and A Plea. Knowledge and Innovation. Journal of the KMCI, 1 , 27-54.

Carrillo, F. J. (2002). Capital Systems: Implications for a Global Knowledge Agenda. Journal of Knowledge Management, 6 (4).

Center for Knowledge Systems (1998). Core KM Processes. Available www.knowledgesystems.org

De Gortari, P. A. (2007). Servicio cívico y voluntariado en Latinoamérica y el 
Caribe Clayss- Vosesa-CSD-ICP, 2 (12).

Mertins, K., Heising, P. \& Vorbeck, J. (Eds.). (2003). Knowledge Management: Concepts and Best Practices (2nd ed.). Berlin Heidelberg, NY: Springer-Verlag.

Torres, C. (2002). Transformación estratégica de un centro de investigación en una organización basada en conocimiento. Panel:
Desarrollo de la Actividad Profesional en Gestión del Conocimiento y la Innovación. Memorias del Congreso Nacional de la ADIAT 2002, México: ADIAT.

Villarreal, H. (2002). Sistema de Capitales en una organización gubernamental. XXXII Congreso de Investigación y Extensión del Sistema Tecnológico de Monterrey, Monterrey. 
RECIE. Revista Electrónica Científica de Investigación Educativa

Vol. 1, núm. 1, enero-diciembre 2012, pp. 211-221.

Martínez-López, M.; Parada Sarmiento, G.; y Madriz Cano, G. 